\title{
Does a dedicated discharge coordinator improve the quality of hospital discharge?
}

\author{
Anita Houghton, Ann Bowling, Kim D Clarke, Anthony P Hopkins, Ian Jones
}

\begin{abstract}
Objective-To evaluate the effectiveness of the role of a discharge coordinator whose sole responsibility was to plan and coordinate the discharge of patients from medical wards.

Design-An intervention study in which the quality of discharge planning was assessed before and after the introduction of a discharge coordinator. Patients were interviewed on the ward before discharge and seven to 10 days after being discharged home.

Setting-The three medical wards at the Homerton Hospital in Hackney, east London.

Patients-600 randomly sampled adult patients admitted to the medical wards of the study hospital, who were resident in the district (but not in institutions), were under the care of physicians (excluding psychiatry), and were discharged home from one of the medical wards. The sampling was conducted in three study phases, over 18 months.
\end{abstract}

Interventions-Phase I comprised baseline data collection; in phase II data were collected after the introduction of the district discharge planning policy and a discharge form (checklist) for all patients; in phase III data were collected after the introduction of the discharge coordinator. Main measures-The quality and outcome of discharge planning. Readmission rates, duration of stay, appropriateness of days of care, patients' health and satisfaction, problems after discharge, and receipt of services.

Results-The discharge coordinator resulted in an improved discharge planning process, and there was a reduction in problems experienced by patients after discharge, and in perceived need for medical and healthcare services. There was no evidence that the discharge coordinator resulted in a more timely or effective provision of community services after discharge, or that the appropriateness or efficiency of bed use was improved.

Conclusions-The introduction of a discharge coordinator improved the quality of discharge planning, but at additional cost.

(Quality in Health Care 1996;5:89-96)

Keywords: discharge coordinator

Effective communication between professionals at the primary-secondary care interface is crucial for good quality inpatient care and timely discharge from hospital. Good quality hospital discharge can be defined as patient satisfaction with involvement in the process of discharge, the absence of problems after discharge and the assessment, documentation, and meeting of the need for community care after discharge.

A recent report from the National Health Service (NHS) Executive recommended that discharge coordinators should be appointed in hospitals to facilitate discharge planning. ${ }^{1}$ An effective discharge planning process may be described as the construction and implementation of a planned programme of continuing care which meets a patient's needs after discharge from hospital. An adequate system of assessing patients' needs should also maximise the efficient use of hospital beds by ensuring that patients are not in hospital for an inappropriate time, or discharged with inadequate notice for the organisation of their continuing care at home. Essential elements of effective discharge planning are a multidisciplinary approach, early and coordinated assessment of a patient's needs and home circumstances, early planning of needs for further care, and effective communication. There is some evidence that the strict application of criteria for inpatient stays, reviewed daily by medical staff for each patient, can reduce the duration of stay. ${ }^{2}$

Although the NHS Executive recommended the appointment of discharge coordinators, there are few supportive publications on the need for the employment of additional professionals to facilitate discharge. The information on the process of discharge and on discharge planning is mainly descriptive, ${ }^{34}$ although one quantitative study has recently been published. ${ }^{5}$ In general, the publications concentrate on people aged over 60 - an important group in view of their greater needs for aftercare from health and social services and their higher risk of readmission. ${ }^{6}$ Apart from elderly people, other groups at risk of readmission are those with chronic illnesses and those who have had two or more surgical procedures performed during the original admission. ${ }^{7}$ Social circumstances are poor predictors of risk of readmission. ${ }^{6}{ }^{7}$ A review of the earlier publications from the United Kingdom showed that thorough preparation of the patient for discharge and discharge planning seemed to be rare; patients were discharged before their community services had been mobilised and there was often inadequate notice of discharge for the patient. ${ }^{8}$ According to a recent review of the situation in Britain, 
little has changed since. ${ }^{9}$ A review of discharge planning in the United States reported that several untested assumptions underlay approaches to discharge planning, including that it is more effective with interdisciplinary input and a designated professional who assumes primary responsibility for coordinating the discharge plan. This was shown by one of the few evaluative studies, conducted by Mamon et al. ${ }^{10}$ They studied 919 admissions of people aged 60 and over to five hospitals in the United States. Patients were interviewed two weeks after discharge. They reported that only when a formal discharge planning case manager was involved in the discharge was there a significant reduction in unmet treatment needs. The results of a recent randomised trial from the United States reported that a discharge planning intervention resulted in fewer readmissions, fewer total days in hospital, lower readmission charges, and lower charges for the healthcare services after discharge. ${ }^{5}$

In the United States, discharge planning has been in operation since the introduction of the prospective payment system (PPS) which has been in effect in most states since 1983. In Britain, the introduction of the NHS and Community Care Act 1990 in April 1993 brought about changes in responsibility and referral practices for managed care in the community. ${ }^{11}$ Also, Department of Health guidelines on the discharge of patients from hospital, ${ }^{12}$ a fuller workbook, ${ }^{13}$ and the introduction of the Patient's Charter ${ }^{14}$ have all focused attention on the need to develop and initiate discharge plans that are appropriate, adequate, based on need, and cost effective. This has been given impetus by the proposed changes in the primary-secondary care interface, particularly in London. ${ }^{15-18}$ As more importance is placed on the significance of planning a quality service that reflects the needs of consumers as well as providers and purchasers, it becomes increasingly necessary to evaluate the effectiveness of discharge planning in the United Kingdom.

Our aim, therefore, was to evaluate the effectiveness of: (a) the implementation of a discharge planning policy; and $(b)$ the role of a discharge coordinator whose sole responsibility was to plan and coordinate the discharge of patients from medical wards. The hypothesis was that such a person would improve the quality of discharge planning and increase the efficiency of bed use compared with that achieved under the existing multidisciplinary arrangements.

\section{Study, design, subjects, and methods}

The study was conducted on all three medical wards at the Homerton Hospital in east London. An intervention study was designed which was carried out in three consecutive phases over a period of 18 months. Phase I (three months) just preceded the introduction of a district's discharge planning policy by nurse managers ${ }^{19}$ and provided baseline data. During this phase there were no formalised ward arrangements for discharge planning. Phase II (next three months) was carried out once the policy had had time to take effect and included the introduction, with the agreement of nurse managers, of a discharge planning checklist, which was to form the basis of the discharge coordinator's planning process - for example, services received before the admission which need restarting; new services needed; functional ability; medication. The policy was simply a restatement of good practice but without giving any one professional responsibility for planning the discharge. It was important that the checklist was introduced at this point so that any improvements occurring as a result of a newly imposed structure on the discharge planning process could be distinguished from those occurring due to the discharge coordinator. Phase III (three months) immediately followed the introduction of the discharge coordinator who had responsibility for assessing patients' needs before discharge (according to the checklist) and to act as coordinator to mobilise services when other staff had not done so. The discharge coordinator was an experienced and fully qualified ward nurse, who was briefed by the research team on her role (based on earlier publications).

The discharge coordinator's role was to make an initial assessment of each patient's needs, to liaise with medical and nursing staff about discharge, ensuring assessment by other healthcare staff and social workers when necessary, to liaise with community services and general practitioners, and to attend ward discharge meetings. During her induction meeting she met all nursing, medical, and other healthcare staff on the participating wards, and with relevant community staff. General practitioners (GPs) were consulted about her new role. The discharge coordinator was entirely hospital based.

All patients admitted to the medical wards at the Homerton hospital were eligible for inclusion providing that they were resident within the boundaries of the local health authority; had not been admitted from an institution; were 18 years of age or older; were under the care of physicians (excluding psychiatrists); and were discharged home from one of the medical wards. Data were collected from patients and their medical records before and after discharge. The aim was to obtain 200 patient responders at each phase. To ensure that sufficient numbers were recruited, patients were initially over sampled to allow exclusion of patients who did not satisfy the sampling criteria (those who transferred to surgical wards) and who did not respond (due to deaths, moves outside the area, refusals). Sampling involved randomly recruiting every third patient who arrived on the wards, in strict chronological order. The sampling and interviewing on the wards and in patients' homes were carried out by a trained researcher, with the help of two interviewers. Two questionnaires were given to each patient, one when the patient was in hospital, and one seven to 10 days after discharge. The questionnaires covered sociodemographic characteristics, health and functional ability, community 
services needed and received (before admission and after discharge), other discharge needs, patients' knowledge and understanding of their condition and treatment, and patients' satisfaction with the discharge process. These items were taken from previous survey questions developed by us. Information was collected from patients' medical and nursing notes on the last day of care, on the reasons for the admission, and on records of assessment of discharge needs and action taken (dichotomised codings of recorded or not recorded for performance of key activities of daily living, need for health and social services, communication with key people for transportation and care after discharge, lay and medical care, communication with the patient about aftercare, and medication). The information required was on procedures and needs, and when discharge needs were not recorded in patients' notes this was coded as such and used as a variable in the evaluation. The questionnaire was based on a literature review of relevant studies, and on previously developed questions. ${ }^{7}$ The questionnaires used were structured in design, with space for any comments.

Most patients completed several questions that measured health status, which were only used to check on any differences in the health of patients which might affect the study results, and to see if the discharge coordinator affected the outcome (she did not). The measurement scales used included the abbreviated mental test, ${ }^{20}{ }^{21}$ the Barthel index, ${ }^{22}$ the RAND SF$36,{ }^{23}$ the RAND short depression screener, ${ }^{24}$ the affect balance scale, ${ }^{25}$ and the appropriateness evaluation protocol. ${ }^{26}$ Detailed results from these scales are documented in the report of the study, available from the authors. ${ }^{27}$ Analyses were carried out with SPSS software, and the tests for significance were $\chi^{2}$ (all two tailed).

Differences reported in this paper were significant, unless otherwise stated. The minimum level of significance taken was $P<0.05$, although most levels were $P<0.001$. The results from each phase are reported separately (there were no differences between the phases for sociodemographic data). Totals do not always equal 626 due to non-response of single items.

\section{Results}

A sample of 600 patients was aimed for. The basis for calculating statistical power is as follows:

With $95 \%$ confidence and $80 \%$ power, with expected patient satisfaction levels of $75 \%$, a sample size of 200 in each group should detect a change of $17 \%$. If the initial patient satisfaction level was $70 \%$ then 200 in each group would detect an increase of $14 \%$. A $15 \%$ increase in patient satisfaction, assuming phase I levels of satisfaction to be $75 \%$, would require two groups of 162 . If we assume an SD of 10 and assuming normality in data on duration of stay, then 200 in each group would detect a difference of three days.

A complete set of information was obtained for a total of 626 patients, 215 in phase I, 204 in phase II and 207 in phase III.
PATIENTS

There were no differences between the phases in sociodemographic characteristics, overall health status, or use of services by the patients before admission. Of the sample $307(52 \%)$ were women; $479(77 \%)$ were aged 55 or over; $280(45 \%)$ were married or cohabiting; $360(59 \%)$ were white European, and $130(21 \%)$ were black (African, Caribbean, or British); 363(59\%) lived in council owned accommodation; 228(38\%) lived alone; $166(25 \%)$ were in paid work; $76(13 \%)$ were in socioeconomic groupings I or II, $92(15 \%)$ in III non-manual, $207(34 \%)$ in III manual, $193(32 \%)$ in IV and V, and $37(6 \%)$ were in other categories; $154(25 \%)$ had received some further education.

\section{CURRENT ADMISSION}

Of the patients studied $569(91 \%)$ were admitted as emergencies. They were under the care of 11 consultants, with $70 \%$ under the care of four of these. They were almost equally divided between the three medical wards. In phase I $74(35 \%)$ patients, in phase II $72(36 \%)$, and in phase III $84(42 \%)$ had been previously admitted to medical wards within the past year. For $31(48 \%)$ of these in phase I, $30(46 \%)$ in phase II, and $31(38 \%)$ in phase III, the reason for that admission was not related to the current admission.

APPROPRIATENESS OF ADMISSION AND DAYS OF CARE

The appropriateness evaluation protocol ${ }^{26}$ was used to assess appropriateness of admission for a sample of the patients. For the current admission, $131(74 \%)$ in phase $\mathrm{I}, 141(70 \%)$ in phase II, and $125(64 \%)$ in phase III were assessed as appropriately admitted. Of the inappropriate cases, $16(8 \%), 12(20 \%)$, and $21(29 \%)$ in phases I, II, and III could have been cared for at home, $8(24 \%), 8(13 \%)$, and $7(10 \%)$ as outpatients, and $18(29 \%), 40(67 \%)$, and $44(61 \%)$ in a non-acute bed. There were no associations between appropriateness of admission and health status. Appropriateness of days of care (assessed during the full 24 hours preceding the day of discharge) varied from $50(29 \%)$ in phase I, $85(42 \%)$ in phase II, and $50(29 \%)$ in phase III. There were no significant differences in appropriateness between the phases (of either admission or days of care).

DURATION OF STAY

The mean (SD, median) duration of stay for phase I was $10 \cdot 55(11 \cdot 35,6)$, for phase II it declined to $7 \cdot 89(8 \cdot 36,6)$, but for phase III it increased to $13 \cdot 52(37 \cdot 78,7 \cdot 00)$. The differences between means for phases I and II and II and III were significant (two tailed $t$ test $2.61, \mathrm{P}<0.01$ for phase I and II; two tailed $t$ test $2.05, \mathrm{P}<0.05$ for phases II and III; differences between phases I and III were not significant). An examination of durations of stay for all patients (including non-study patients) admitted to medical wards at Homerton during the same period showed no such difference between the phases. 
DISCHARGE PL ANNING PROCESS

There were no differences between the three groups in how much notice they had been given of their discharge, their views on their duration of stay, how much information they had on their illness, and medication before they were discharged. In phase I $45(23 \%)$, in phase II $61(31 \%)$, and in phase III $51(25 \%)$ had less than six hours or no notice of their discharge, but $175(88 \%)$ in phase I, $178(90 \%)$ in phase II, and $186(91 \%)$ in phase III said that they had enough time to make their arrangements. Between $34(17 \%)$ in phase I, $41(21 \%)$ in phase II, and $41(20 \%)$ in phase III thought that their duration of stay was too short. In the three phases $173(85 \%), 172(86 \%)$, and $189(89 \%)$ said that they would have liked more information about their discharge, for most, on their medical condition. In phases II $(160(80 \%))$ and III $(153(74 \%))$ patients were less likely to have been given a discharge date than patients in phase I $(187(93 \%))$, but patients in phase III $(140(91 \%))$ were significantly more likely to have been discharged on that date than in phases I $(143(76 \%))$ and II $(126(82 \%)$ ) (tables 1 and 2$)$. There were no differences with age. Of the patients in phase III $130(64 \%)$ were asked in detail about their home circumstances compared with $74(36 \%)$, and $77(40 \%)$ in phases I and II $\left(\chi^{2} 17 \cdot 08\right.$; $\mathrm{P}<0.001)$. Patients in phase III were twice as likely to have been visited by a social worker in hospital than the patients in the other two groups $-62(30 \%)$ patients in phase III com-

Table 1 Data on discharge date given

\begin{tabular}{lccc}
\hline $\begin{array}{l}\text { Were you given a definite } \\
\text { discharge date? }\end{array}$ & $\begin{array}{c}\text { Phase I } \\
n(\%)\end{array}$ & $\begin{array}{c}\text { Phase II } \\
n(\%)\end{array}$ & $\begin{array}{c}\text { Phase III } \\
n(\%)\end{array}$ \\
\hline Yes & $187(93)$ & $160(80)$ & $153(74)$ \\
No & $15(7)$ & $39(20)$ & $53(26)$ \\
Total & 202 & 99 & 206
\end{tabular}

Overall $\chi \vdots 24 \cdot 32 ; \mathrm{P}<0 \cdot 001$

Linear trend $x \div 23.48 ; P<0.0001$

Departure from linear trend $x \div 0.84 ; P=0.36$

Subscrinted numbers are degrees of freedom.

Table 2 Data on actual discharge date

\begin{tabular}{lccc}
\hline $\begin{array}{l}\text { If ves, was this the date you } \\
\text { were actually discharged? }\end{array}$ & $\begin{array}{c}\text { Phase I } \\
n(\%)\end{array}$ & $\begin{array}{c}\text { Phase II } \\
n(\%)\end{array}$ & $\begin{array}{c}\text { Phase III } \\
n(\%)\end{array}$ \\
\hline Yes & $143(76)$ & $126(82)$ & $140(91)$ \\
No & $44(24)$ & $27(18)$ & $14(9)$ \\
Total & 187 & 153 & 154 \\
\hline
\end{tabular}

Overall $\chi \div 12 \cdot 39 ; \mathrm{P}<0.01$

Linear trend $\chi_{i}^{2} 12 \cdot 26 ; P<0 \cdot 0005$

Departure from linear trend $x \div 0 \cdot 13 ; P=0.72$

Subscripted numbers are degrees of freedom. pared with $31(15 \%)$ and $27(14 \%)$ patients in phases I and II $\left(\chi^{2} 10 \cdot 17 ; P<0 \cdot 01\right)$. In phases I and II, $11(12 \%)$ and $13(15 \%)$ said that they were able to discuss their anxieties about discharge and aftercare with a member of staff in hospital who was rated as helpful; this increased to $49(88 \%)$ at phase III $\left(\chi^{2} 41.91\right.$; $\mathrm{P}<0.001$ ).

A checklist or clear discharge plan was more likely to have been used in each successive phase (table 3). Patients in phase III were more likely to have documentation about their care arrangements in the hospital records than patients in either of the other two phases (table 3). In phases I, II, and III, 156(75\%), $159(81 \%)$, and $172(84 \%)$ patients said that they had been given a discharge letter to give to their GPs, showing a significant increase between phases I to III - the whole period of the study (phase I and III $\chi^{2} 6 \cdot 80 ; \mathrm{P}<0 \cdot 01$ )

FUNCTIONAL ABILITY, INFORMAL SLPPOR', AND HEALTH

There were no differences between the phases in the overall mental or physical health or functional ability of patients at follow up..$^{20} .25$ most were functionally able. Long term feelings of depression and negative moods were evident with both the RAND scales and the affect balance scale. Altogether 63(32\%) patients in phase I, 64(33\%) in phase II, and $70(36 \%)$ in phase III said, in response to a RAND depression screening item, that they felt depressed or sad most days in the past two years.

PROFESSIONAL SERVICES RECEIVED ANI) PROBIEMS ENCOUNTERED

Only $4(2 \%)$ patients in phase III had problems with hospital transport on discharge, in comparison with $5(10 \%)$ patients in phase II, and $17(17 \%)$ patients in phase I (phases I and II $\chi^{2}$ 9.34; $\mathrm{P}<0.01$; phases II and III $\chi^{2} 1.31$; non-significant; phases I and III $X^{2} 7 \cdot 65$; $\mathrm{P}<0.01$ ).

Patients in phase III were significantly less likely to have been visited by a GP since discharge $(1(1 \%))$ than patients in either phase II $(10(6 \%))$ or phase I $(39(20 \%))$ (phases I and II, $X^{2} 19.33 ; \mathrm{P}<0.001$; phases II and III $\chi^{2} 7.91 ; \mathrm{P}<0.01$; phases I and III $\chi^{2} 37.99$; $P<0.001)$. There was little difference between phases in the receipt of other services. Patients were asked if the health and social services professionals allocated had arrived when

Table 3 Data on discharge plan or check list and documentation of arrangements for aftercare

\begin{tabular}{|c|c|c|c|c|c|c|}
\hline & \multicolumn{3}{|c|}{ Has a discharge plan or chicklist been used? } & \multicolumn{3}{|c|}{$\begin{array}{c}\text { Haie arrangements for after care been documemed by } \\
\text { staff? }\end{array}$} \\
\hline & $\begin{array}{l}\text { Phase I } \\
n(\% / 11)\end{array}$ & $\begin{array}{c}\text { Phase II } \\
n(\%)\end{array}$ & $\begin{array}{l}\text { Phase III } \\
n(\%)\end{array}$ & $\begin{array}{l}\text { Phase I } \\
n(\%)\end{array}$ & $\begin{array}{l}\text { Phase II } \\
n(\%)\end{array}$ & $\begin{array}{l}\text { Phase III } \\
\text { n(") }\end{array}$ \\
\hline Yes & $2(1)$ & $124(62)$ & $203(99)$ & $37(19)$ & $40(20)$ & $81(40)$ \\
\hline No & $199(99)$ & $77(38)$ & $3(1)$ & $162(81)$ & $160(80)$ & $124(60)$ \\
\hline Total & 201 & 201 & 206 & 199 & 200 & 205 \\
\hline
\end{tabular}

Discharge plan used:

Overall $x^{2}=396 \cdot 8 ; P<0.001$

Linear trend $\chi^{2} 389 \cdot 11 ; \mathrm{P}<0.0001$

Departure from linear trend $X^{2} 7.7 ; P=0.0055$

Departure from linear trend
Arrangements made:
Overall $\chi^{2} 28.75 ; \mathrm{P}<0 \cdot 001$.

Linear trend $\chi_{i}^{2} 23.07 ; \mathrm{P}<0.000$

Departure from linear trend $x^{2}+5 \cdot 68 ; P=0.0172$ 
Table 4 Data on aftercare services

\begin{tabular}{lc}
\hline $\begin{array}{l}\text { For those expecting services, did they } \\
\text { arrive on the day expected? }\end{array}$ & $n(\%)$ \\
\hline General practitioner: & \\
Yes & $27(63)$ \\
No, late & $11(26)$ \\
Not arrived yet & $4(9)$ \\
No, early & $1(2)$ \\
District nurse: & $18(30)$ \\
Yes & $33(55)$ \\
No, late & $5(8)$ \\
Not arrived yet & $4(7)$ \\
No, early & \\
Meals on wheels: & $9(53)$ \\
Yes & $6(35)$ \\
No, late & $2(12)$ \\
Not arrived yet & $0(0)$ \\
No, early & \\
Home help: & $30(51)$ \\
Yes & $21(36)$ \\
No, late & $3(5)$ \\
Not arrived yet & $5(8)$ \\
No, early & $17-60$ \\
Number of respondents &
\end{tabular}

expected. Table 4 shows that most GPs arrived on the day they were expected, $26 \%$ arrived on a day later than this, and $9 \%$ had not arrived; just $30 \%$ of district nurses and $53 \%$ of meals on wheels arrived when expected. There were no differences between phases, and the discharge coordinator had not led to any improvements in the speed with which services were (re)started.

PROBLEMS AFTER DISCHARGE

Patients in phase III were less likely than those in phases I and II to have failed to receive any prescribed treatment or care (table 5). Fewer patients in phase III $(26(13 \%))$ than in phases I $(58(28 \%))$ and II $(45(23 \%))$ reported experiencing complications from their treatment in hospital which hospital staff did not warn them about (phase I and II $=\chi^{2} 1 \cdot 88$; nonsignificant; phases II and III $=\chi^{2} \quad 7 \cdot 38$; $\mathrm{P}<0.01$; phases $\mathrm{I}$ and $\mathrm{III}=\chi^{2} \quad 16.59$; $\mathrm{P}<0.001)$. Patients in phases II $(114(59 \%))$ and III $(125(63 \%))$ were significantly less likely to have reported the need to see the hospital doctor again than patients in phase I $\left(159(78 \%)\right.$ ) (phases I and $I I=\chi^{2} 16 \cdot 30$, $\mathrm{P}<0.001$; phases II and III $=\chi^{2} 0.90$, not

Table 5 Problems experienced after discharge

\begin{tabular}{|c|c|c|c|}
\hline Problem & $\begin{array}{c}\text { Phase I } \\
n(\%)\end{array}$ & $\begin{array}{c}\text { Phase II } \\
n(\%)\end{array}$ & $\begin{array}{c}\text { Phase III } \\
n(\%)\end{array}$ \\
\hline \multicolumn{4}{|l|}{$\begin{array}{l}\text { Patient failed to receive } \\
\text { treatment care or prescribed } \\
\text { medication that the hospital } \\
\text { staff advised him or her to get:* }\end{array}$} \\
\hline $\begin{array}{l}\text { No } \\
\text { Yes }\end{array}$ & $173(85)$ & $172(89)$ & 197(96) \\
\hline \multirow{3}{*}{\multicolumn{4}{|c|}{$\begin{array}{l}\text { Patient experienced problems } \\
\text { or complications arising from } \\
\text { treatment in hospital which } \\
\text { staff did not warn about: } \dagger\end{array}$}} \\
\hline & & & \\
\hline & & & \\
\hline Yes & $58(28)$ & $45(23)$ & $26(13)$ \\
\hline & & & $180(87)$ \\
\hline \multicolumn{4}{|l|}{$\begin{array}{l}\text { Patient felt the need to see } \\
\text { the hospital doctor again: }\end{array}$} \\
\hline Yes & $159(78)$ & $114(59)$ & $125(63)$ \\
\hline & $46(22)$ & $80(41)$ & $72(36)$ \\
\hline Number of respondents & $200-205$ & $193-196$ & $197-206$ \\
\hline \multicolumn{4}{|c|}{$\begin{array}{l}\star \text { Overall } \chi_{2}^{2} 12 \cdot 61 ; \mathrm{P}<0 \cdot 01 \text {. } \\
\text { Linear trend } \chi_{1}^{2} 12 \cdot 36 ; \mathrm{P}<0 \cdot 0004 . \\
\text { Departure from linear trend } \chi_{1}^{2} 0 \cdot 25 ; \mathrm{P}=0.62 \text {. } \\
\text { †Overall } \chi_{2}^{2} 16 \cdot 57 ; \mathrm{P}<0 \cdot 001 \text {. } \\
\text { Linear trend } \chi_{1}^{2} 16 \cdot 21 ; \mathrm{P}<0 \cdot 0001 \text {. } \\
\text { Departure from linear trend } \chi_{1}^{2} 0 \cdot 36 ; \mathrm{P}=0.55 \text {. } \\
\text { fOverall } \chi_{2}^{2} 17 \cdot 34 ; \mathrm{P}<0 \cdot 001 . \\
\text { Linear trend } \chi_{1}^{2} 9 \cdot 21 ; \mathrm{P}<0 \cdot 0002 . \\
\text { Departure from linear trend } \chi_{1}^{2} 8 \cdot 13 ; \mathrm{P}=0.004 \text {. } \\
\text { Subscripted numbers are degrees of freedom. }\end{array}$} \\
\hline
\end{tabular}

significant; phases I and III $=\chi^{2} \quad 9 \cdot 64$, $P<0.01)$.

\section{PATIENT SATISFACTION}

In phase I $153(76 \%)$ respondents, in phase II $155(84 \%)$, and in phase III $186(90 \%)$ reported that they were very satisfied or satisfied with the arrangements made for their discharge. This increase in patient satisfaction about discharge was significant between phases I and III $\left(\chi^{2} 8 \cdot 46 ; P<0.01\right)$, not between II to III $\left(\chi^{2}\right.$ $0 \cdot 49$; non-significant), and between I and III $\left(x^{2} 14.01 ; P<0.001\right)$. There was a similar trend in patients' reports of their carers' satisfaction, although this did not reach significance.

READMISSION RATES

There were no significant differences in readmission rates (within seven days of discharge) between the three phases. For phases I, II, and III the readmission rates were $18(9 \%), 21(11 \%)$, and $15(7 \%)$, and eight of these people had been readmitted more than once. Readmission rates within 28 days of discharge for all patients during the same periods dropped from $2.6 \%$ in phase I, to $2 \cdot 1 \%$ in phase II, and $1.7 \%$ in phase III.

\section{Discussion}

Good quality hospital discharge can be defined in relation to timely discharge, patient satisfaction with the process of discharge and communication about it, the absence of problems after discharge, and the assessment, documentation, and meeting of need for community care from health or social services after discharge.

The results of this study indicate that the employment of a discharge coordinator results in an improved discharge planning process, and reduces patients' problems after discharge. However, the discharge coordinator had no impact on the more timely or effective provision of community services after discharge, and did not improve the appropriateness or efficiency of bed use.

On the positive side, patients in phase III were significantly more likely to have been asked about their home circumstances while they were in hospital than those in either of the other two phases and they were twice as likely to have been visited by a social worker. They were also three times as likely to have reported that they were able to discuss their anxieties about their discharge with a member of staff, and significantly more likely to have had documentation about their arrangements after discharge in the medical notes. For all these factors, there was no significant difference between phases I and II, which strongly suggests that improvements were due specifically to the discharge coordinator, rather than improvements in discharge planning generally. On the the other hand, there were some areas in which improvements took place gradually over the whole period of the study or in which the main improvements took place between phases I and II. For example, there was a significant decrease between all the phases in the proportion of patients who had been visited 
by their GP in the period after discharge and a decrease in the proportion of patients who said that they felt the need to see the hospital doctor again. These findings are reflected in the lower proportion of the patients in phase III reporting problems relating to their admission that they had not been warned about, and reporting failure to receive prescribed treatment or care. Although these factors showed a trend over the three phases, the significant differences occurred between phases II and III, rather than I and II.

There are therefore two sets of effects that need to be disentangled. Firstly we must distinguish between those improvements occurring as a result of improved discharge planning after the implementation of the local policy and those occurring as a result of the introduction of the discharge coordinator. Secondly, it is important to distinguish between those areas in which the discharge coordinator seemed to be effective and those in which she did not.

For changes in discharge planning, there is no doubt that there were some general improvements over the whole period of the study which were probably due to a combination of the implementation of the local policy developed jointly by those working in health and social services, the introduction of the discharge checklists, and a general increase in awareness about discharge planning caused by the study itself. However, it is clear that the greatest improvement occurred after the introduction of the discharge coordinator, strongly suggesting that this post had a beneficial effect. This effect, however, was not universal, but seemed to be confined to those matters concerning the medical welfare of the patient and the successful arrangement of medical and other - for example transport - services that are usually the responsibility of ward staff. The discharge coordinator seemed to have little effect on the efficiency with which community services were provided or whether they were provided at all. This highlights the limitations of such a post, and shows that for discharge planning to be successful it has to be combined with the adequate provision of effectively organised community services.

An effect essential to make the idea of a discharge coordinator appeal to NHS managers is an increase in efficiency of use of hospital beds. To assess the extent to which this was a reality, we considered three indicators of efficiency: duration of stay, appropriateness of the day preceding discharge as assessed by the appropriateness evaluation protocol,,$^{26}$ and readmission rates.

For duration of stay, the introduction of the discharge coordinator was actually associated with an increase. A problem caused by the longitudinal design of this study is that it is not always possible to conclude that any changes that occurred did so as a result of the intervention, as opposed to other factors which may play a part, but are not obvious. To examine the possibility that something other than the discharge coordinator had produced an increase in duration of stay, data for all patients admitted during the three study periods were extracted from routine hospital sources. It was found that no increase in duration of stay had taken place over the study period, suggesting that the work of the discharge coordinator was the important variable in slightly increasing stays between phase II and phase III. It seems that the introduction of the checklist in phase II may have had the earlier effect of reducing duration of stay, whereas the further intervention of human decision making (the discharge coordinator) in effect negated this and increased the duration of stay.

In the United States, hospital managers have been sufficiently convinced that discharge coordinator's carry out good quality discharge planning and that they improve the efficiency of bed use and are therefore cost effective. Such posts have been introduced in many hospitals. We think that although the United Kingdom should seriously consider any measure that improves quality and efficiency, it is important that it is not introduced without proper evaluation.

More patients in phase III saw a social worker. It is possible that the recommendations of a social worker might have masked a reduction in duration of stay. Patients in phase III were also less likely to see a GP after discharge, implying that their process of discharge obviated any need for this.

Moving on to appropriateness of days of care, appropriateness rates were very low, especially in phases I and III. This is because the day of care was taken to be the full day preceding the day of discharge and it is known from previous studies using the appropriateness evaluation protocol, ${ }^{20}$ that the closer a day is to the patient's discharge the less appropriate it is likely to be. It is impossible to say why appropriateness rates were so much higher for phase II. This may have been due either to natural variation or perhaps an unusual increase in pressure on beds at that time. What is clear is that the discharge coordinator did not have any beneficial effect on appropriateness of bed use.

Finally, we looked at readmission rates as they may act as indicators of appropriateness and quality of discharge. No changes were found in readmission rates, although there was a decrease for all patients admitted during the same periods. The Philadelphia study on discharge planning ${ }^{5}$ was published after the analysis of our data, and in the course of preparation of this paper. This group reported that the introduction of a discharge planning protocol (not planner) had no effect on the initial duration of stay $(7 \cdot 4-7 \cdot 5$ days for a similar elderly population) but the chances of hospital readmission within 14 days were significantly less in the intervention group ( $3 / 72$ as opposed to $11 / 70$ ). One possible explanation for at least the part of the group differences in the United States study is that randomisation resulted in an excess of men in the intervention group ( $\mathrm{M}: \mathrm{F}$ intergroup ratio $1 \cdot 4: 1$ ), and it is known that male sex is more strongly associated with readmission, ${ }^{27}$ perhaps reflecting different expectations between the sexes. The study cited by Weissman and colleagues ${ }^{2 *}$ shows how 
difficult it would be to use only readmission rates as a measure of success of discharge planning. Income, social class, and home ownership were all associated with readmission rates in their study, yet these data items are not routinely captured by health services.

It is difficult to analyse the costs and benefits of the introduction of a discharge coordinator as so many factors are involved. This would necessitate the estimation of costs saved through lower consultation rates with GPs, lower hospital doctor consultation rates, possibly lower readmission rates (and lower use of community services for those whose discharge was delayed), and the benefits of freeing nurses time by relieving them of some of the responsibility for discharge planning. It is difficult to express in monetary terms the benefits of avoiding problems after discharge, increasing patient satisfaction, increasing compliance in treatment, and reducing problems with transport. Added to these problems are the difficulties in distinguishing the costs that would be saved by the hospital from those saved by primary care and community services. Hospital managers are unlikely to be very concerned about the costs of community services, given the way that health care is funded at present in the United Kingdom. In different systems of health and social care however, useful cost savings may result, at least for the hospital. ${ }^{5}$

The costs of the discharge coordinator, except for salary, are equally difficult to estimate. There is the increase in duration of stay in our study but this cannot be simply estimated from average costs per bed day as costs fall the further a patient is into an admission. There are the extra costs resulting from an increased referral rate to social workers and health professionals, an increase in paperwork, numbers of telephone calls, and time taken to liaise with colleagus. The one cost which is simple to estimate is the salary of the discharge coordinator, which was nursing grade $\mathrm{F}$. The cost per patient discharged was $£ 21$ on the basis of the discharge coordinator assessing and planning the discharge for an average of 14 patients a week.

The ideal design for an intervention study of this kind would be a randomised controlled trial - that is, random allocation of patients into two groups in which one group would receive the intervention, in this case, the services of a discharge coordinator, and the other would not. However, we considered that there were some serious and insurmountable problems associated with this approach. Firstly, the random selection of patients would mean that those receiving intervention would often be situated in the wards next to controls. With no control over contact between these patients and between controls and other ward staff, "contamination" would be inevitable. Also, the presence of a discharge coordinator on the ward, a major part of whose job is to liaise with all staff involved with discharging patients, would undoubtedly result in a Hawthorne effect. In other words, discharge planning would improve generally during the period of study.
One possible solution to these problems was to obtain the intervention and control groups from different wards. However, it became clear during the planning phase of the study that standards of discharge planning varied widely between the three wards concerned and could not, therefore, be compared. Even if two wards were selected and discharge planning assessed both before and after the introduction of a discharge coordinator on one of the wards, with the other as the control, it is more than probable that the potential for improvement would be dependent on the standards before intervention and therefore the results would depend on which ward was chosen. It was for these reasons that a randomised control trial was rejected in favour of the intervention study. This study design had several advantages over a randomised controlled trial. There would be no contamination between patients; any general improvement in discharge planning occurring as a result of introducing a discharge coordinator would not affect the results; and the effect of the discharge coordinator could be isolated from the effect of imposing a new structure onto the discharge planning process.

Although the study results might be criticised for being person specific - that is, there was only one discharge coordinator evaluated - and therefore the results are dependent on the proficiency of that person, there are several ways in which the quality of that person's work was monitored - for example, the proportion of cases in which a discharge form was completed, the proportion of patients who were assessed for discharge, and the referral rate to social workers. Such evidence showed the discharge coordinator to be highly efficient and unpublished data from a staff survey showed that she had formed excellent working relations and was reported to be helpful and supportive.

In conclusion, there was evidence that some aspects of the discharge process improved as a result of the implementation of the district's discharge planning policy and the introduction of discharge check lists. These improvements were reflected in a moderate reduction in problems relating to the admission experienced by patients after discharge. The introduction of the discharge coordinator resulted in a further improvement in the discharge planning process, a reduction in problems after discharge, and a reduction in the need for medical and related services. However, there was no evidence that intervention resulted in more efficient or timely provision of services in the community or that improved discharge planning resulted in the improved efficiency of hospital bed use. Indeed, there were indications that the discharge coordinator increased, rather than decreased, duration of stay. There was also no evidence that the discharge coordinator had any effect on the overall physical and emotional outcome of a hospital admission. In summary, the introduction of a discharge coordinator in the United Kingdom improved the quality of discharge planning but at not inconsiderable cost. We would therefore question the recommendation in the recent 
government report that discharge coordinators should be appointed. ${ }^{1}$

Finally, most of the focus of health services research has been on the clinical effectiveness of medical interventions. There has been relatively little attention paid to evaluating methods of service delivery, or to the most appropriate methods of evaluating the effectiveness of organisational change. These gaps are glaring; not surprising, given the difficulties of designing and implementing randomised controlled trials in real life organisational situations and the urgent need for methodological debate. It is hoped that the study presented here will provide an illustration of the difficulties of a randomised controlled trial design, and an example of how alternative designs can provide fruitful data.

We thank the hospital staff and patients for their cooperation with this study, in particular the discharge coordinator, and Lesley Marriott for typing this manuscript. This work was supported by a grant from the Wellcome Trust.

1 NHS Executive. Report on urgent and emergency admissions to hospital Clinical Standards Advisom Group London: HMSO, 1995

2 Mozes B, Halkin H, Schiff E, Modan B. Reduction of redundant hospital stay through controlled intervention. Lancet 1987;i:95-6.

3 Waters KR. Discharge planning: an explanatory study of the process of discharge planning on geriatric wards. F Adz process of discharge
Nurs $1987 ; 12: 17-83$

4 Waters KR. Outcomes of discharge from hospital for elderly people. $\mathcal{F}$ Adv Nurs 1987;12:347-55.

5 Naylor M, Brooten D, Jones R, Lavizzo-Mourey R, Mezey M, Pauly M. Comprehensive discharge planning for the hospitalized elderly: a randomized clinical trial. Ann Intern Med 1994;120:99-1006.

6 Holloway JJ, Vanderbrugh Medendorp S, Bromberg J. Risk factors for early readmission among veterans. Health Ser Res 1990;25:213-37.

7 MaGuire PA, Taylor IC, Stout RW. Elderly patients in acute medical wards: factors predicting length of stav in medical wards: factors predictin
hospital. BMF 1986;292:1251 -3

8 Bowling A, Betts G. Communication on discharge. Nursing Times 1984;80:31-3. 9arks L. Seamless care or patchwork quilt? Discharging paticnts from acute hospital care in the 1990s. London: Kings Fund Institute, 1993

10 Mamon J. Steinwachs DM, Fahey M, Bone LR, Oktay J, Klein L. Impact of hospital discharge planning on meeting patient needs after returning home. Health Sere Res 1992;27:155-75

11 Department of Health. National Health Siricic and Community Care Act. London: HMSO, 1990

12 Department of Health. Discharge of pationts from hospital. London: HMSO, 1989. $(\mathrm{HC}(89) 5$ and summary $\mathrm{LAC}(89) 7$ ).

13 Department of Health. Hospital discharge workbook: a manual on hospital discharge practice. London: HMSO,

14 The Paticut's Charter. London: HMSO, 1991

15 Tomlinson, B. Report of an inquiry into London's health service medical education and research. London: HMSO, 1992

16 Moss F, McNicol M. Secondary care beyond Tomlinson: an opportunity to be seized or squandered. BMF 1992;305:1211-4.

17 Department of Health. Making London bitter. Manchester Publications Unit, 1992.

18 Beardshaw V, Gordon P, Pampling D. Primary care development zones. BM7 1992;306:323-5.

19 The Bart's NHS Trust. St Bartholomes's, Homerton and Hackney Hospitals discharge plaming policy. London: St Bartholomew's Hospital, 1993.

20 Hodkinson HM. Evaluation of a mental test score for the assessment of mental impairment in the elderly. Agc Ageing 1972;1:233-8

21 Jitapunkul S, Pillay I, Ebrahim S. The abbreviated mental test. Age Ageing 1991;20:332-6.

22 Mahoney FI, Barthel DW. Functional evaluation: the Barthel index. Marvland State Medical foumal 1965;14:61-5. (Adapted for self administration by Gompertz P, Ebrahim S, (unpublished, Department of Public Health, Roval Free Hospital Medical School.))

23 Stewart AL, Ware JE. Mcasuring functioning and well-bcing: the medical outcomes study approach. Durham: Duke University Press, 199 ?

24 Burnam MA, Wells K, Leake B, Landsverk J. Development of a brief screening instrument for detecting depressive disorders. Med Care 1988:26:775-89.

25 Bradburn NM. The structure of psichological well-bing. Chicago: Aldine, 1969

26 Gertman PM, Restuccia JD. The appropriateness evaluation protocol. Med Care 1981:19:855-71.

27 Clarke K, Houghton A, Bowling A, Hopkins AP. Eruluation of a discharge planner in medical wards in an inner cit hospital. Report prepared for the Wellicome Trust. London. St Bartholomew's and Royal London Medical Schools and The Royal College of Physicians, London, 1993.

28 Weissman JS, Stern RS, Epstein AM. The impact of patient socioeconomic status and other social factors on readmission: a prospective study from four Massachusetts readmission: a prospective study fr
hospitals. Inquiry 1994;31:163-72. 